\title{
Real time thin-film thickness monitoring sensors: a comparative study of dual mode Solidly Mounted Resonators with commercial Quartz Crystal Microbalances
}

Teona Mirea
Manuel Moreno
Nicolò Chiodarelli
Mario DeMiguel-Ramos

\begin{abstract}
Next generations of physical and chemical vacuum deposition systems require more accurate and faster sensors to monitor the deposition of very thin films ( $\mathrm{nm}$ range). Acoustic sensors, particularly QCMs, have been typically used in such systems. How ever, their potential has reached certain limitations. They are bulky, they need a water-cooling system to work at higher temperatures, and for certain applications their response time is not fast enough. For many years, film bulk acoustic wave resonators (FBARs) working at higher frequencies have been studied as a higher sensitivity alternative to QCMs, particularly in biosensing applications. However, they have been never applied and simultaneously compared to QCMs in an actual PVD system so far. Sorex Sensors has developed an FBAR which not only offers higher mass sensitivity than QCMs but also allows discriminating temperature related effects within the same device without the need of a reference sensor. FBARs can also be integrated in arrays providing a cost-effective and more accurate solution for thin film monitoring. This sensor has been tested simultaneously with a commercial QCM in an evaporator, for benchmarking the characteristic of both technologies in terms of sensitivity.
\end{abstract} film.

Keywords-Solidly mounted resonators, QCM, sensitivity, thin

Quartz crystal microbalances (QCMs) are widely known for their application as thin film thickness monitoring sensors in physical vacuum deposition (PVD) systems [1]. Commercial QCMs work at 5-6 MHz providing mass sensitivities of around $0.2 \mathrm{~Hz} \mathrm{~cm} / \mathrm{ng}[2]$. Considering that the frequency resolution offered by deposition controllers on the market varies from the best available $0.0035 \mathrm{~Hz}$ [3] to $0.03 \mathrm{~Hz}$, one may expect QCMs to provide mass resolutions of around $0.04 \mathrm{ng} / \mathrm{cm}^{2}$ to $0.6 \mathrm{ng} / \mathrm{cm}^{2}$, when coupled to these systems. So far, their performance has been satisfying for many applications. However, scientific advances and new industrial applications, such as Atomic Layer Deposition where thin-films and interfaces are engineered at the molecular level, impose new requirements that actual QCMs can hardly fulfil. As an example, the vertical cavity surface emitting laser (VCSEL) technology is taking off due to the increasing demand in the automotive and the consumer electronics industries [4]. In these fields, VCSELs are being used as optical sensors for autonomous vehicles or for facial recognition in smartphones. Their structure includes Bragg reflectors made of several layers that are few-nm thick. As the accurate thicknesses of the thin films composing the VCSELs is critical, the next generation of deposition systems must provide highly controlled homogeneity and accuracy. Some high-quality tools include optical techniques for thickness monitoring. However, these are bulky, expensive and rely on the refractive index of the deposited materials (i.e. dielectric materials in this case), which can be difficult to measure and calibrate for very thin layers. In this context, solidly mounted resonators (SMRs), or film bulk acoustic wave resonators (FBARs, ) working at $\mathrm{GHz}$ frequencies represent a promising and cost-effective alternative [5], [6]. They use the same acoustic sensing principles as QCMs, but offer a new set of advantages that overcome their limitations. They offer mass resolutions in the fg range, their size can be three orders of magnitude smaller (see Fig. 1), they can also work at very high temperatures [7] and can be integrated in array-based chips using standard MEMS processed. Using an array configuration of SMRs increases accuracy and precision as it allows averaging the input of several sensors undergoing the same deposition conditions. Regarding temperature stability, some quartz cuts have low temperature coefficient of frequency (TCF), but they still require a (water) cooling systems, which increases the size of the sensing system. SMRs have proven to achieve very low TCF values, and although this value is not linear upon deposition of certain materials and thicknesses, it can be predicted, thus compensated. At Sorex Sensors we have developed SMRs that can discriminate the temperature influence from mass sensing without the need of reference devices.

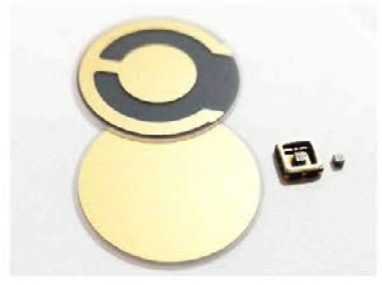

Fig. 1. On the left two commercial $\mathrm{QCM}_{\mathrm{S}}$ and on the right the Sorex Sensors SMR packaged and unpackaged. 
The superior performance in terms of mass sensitivity of FBARs is widely known, however their behavior has never been monitored in real-time while integrated in PVD systems until now. With this work, we present our initial results with SMRs used as thin film monitors in an evaporation system.

\section{EXPERIMENTAL TECHNIQUES}

\section{A. Devices}

Our devices are specifically designed SMRs working at 2.0 $-2.6 \mathrm{GHz}$ with two resonances (Fig. 2). SMRs are based on a piezoelectric material, particularly AlN, sandwiched between two metallic electrodes and mounted on an acoustic reflector to isolate the resonant cavity from the Si substrate (inset Fig. 2). The acoustic reflector consists of alternating layers of high and low acoustic impedance materials. We generate two resonances with different mass sensitivities and different TCFs by engineering the acoustic reflector stack.

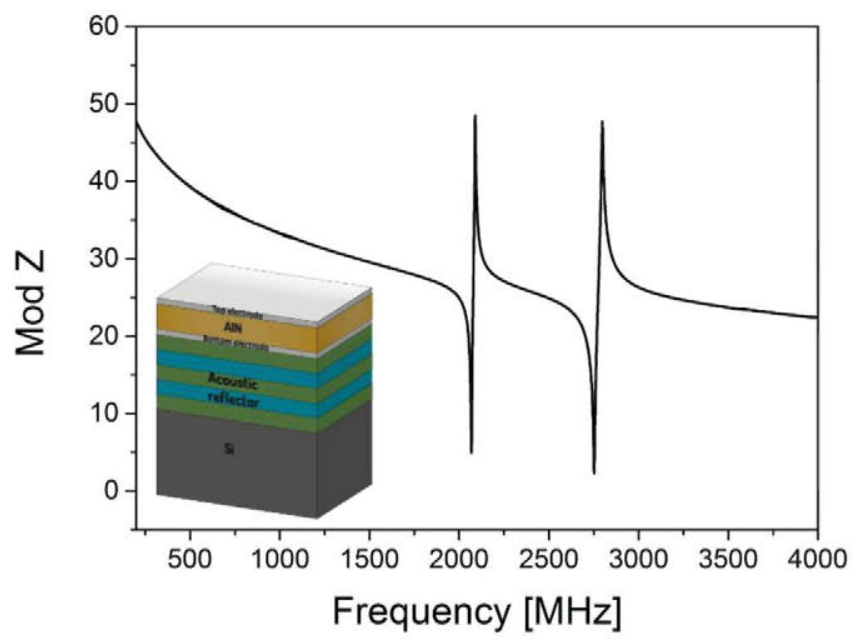

Fig. 2. Structure and response of the SMR, showing peak 1 (around $2 \mathrm{GHz}$ ) and peak 2 (around $2.6 \mathrm{GHz}$ )

\section{B. Set-up}

The sensors have been integrated in an evaporator system together with a commercial $6 \mathrm{MHz}$ QCM, placed at a close distance at the upper side of the chamber (Fig. 3). The deposited material was $\mathrm{SiO}_{2}$ evaporated by Joule effect. Its evaporation rate was controlled using another QCM placed below the shutter. The response of the SMR was monitored using a network analyzer and the one of the QCM using a commercial Inficon STM-2. The temperature of the SMR was measured with a thermocouple. All data were acquired using LabView.
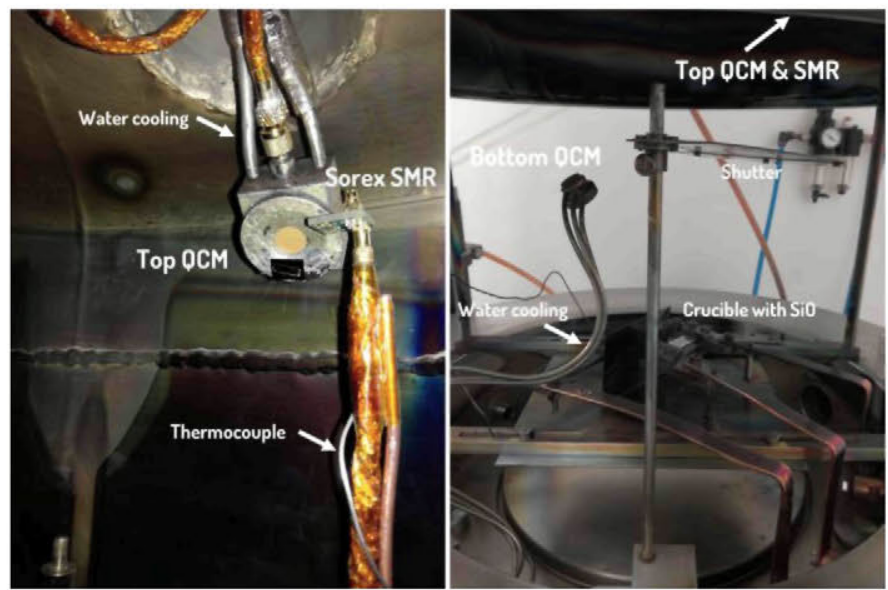

Fig. 3. Set-up inside the evaporator.

\section{RESULTS AND DISCUSSION}

Fig. 4 shows the response of both the SMR and QCM upon deposition of $275 \mathrm{~nm}$-thick $\mathrm{SiO}_{2}$. The graph shows the shifts in frequency of the QCM and the dual resonances of the SMR, together with the temperature variation close to the sensors and the thickness evolution. It can be clearly observed the difference in sensitivity of the SMR resonances, being more sensitive the first one $(2 \mathrm{GHz})$. This also occurs with their TCF, although in this case the second peak shows higher sensitivity to temperature than the first one (see Fig. 5). Having two different responses to mass and temperature within the same device allows to discriminate the temperature effect with a simple set of equations.

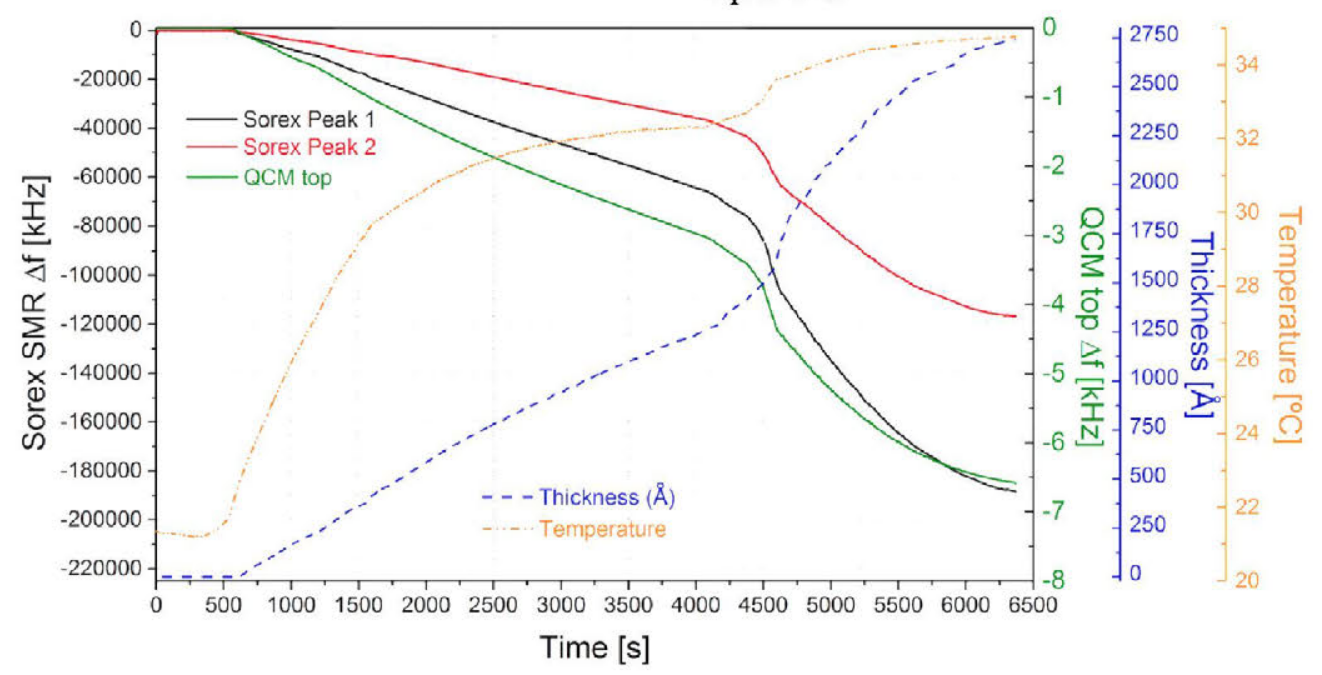

Fig. 4. Frequency response of the Sorex Sensor SMR (two resonances) and the QCM placed close to it 


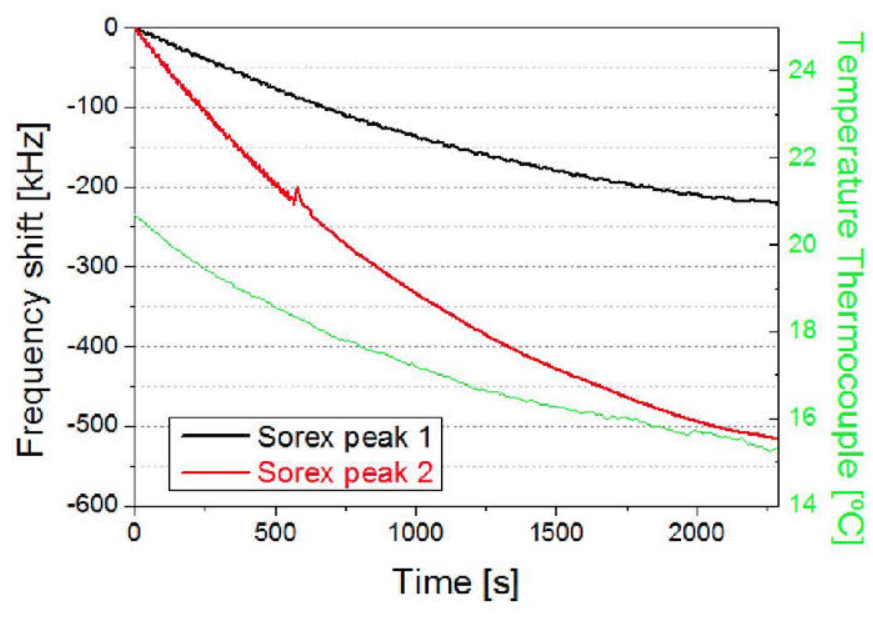

Fig. 5. Shifts in frequency of the SMR resonances with temperature variation.

For a total deposition of $275 \mathrm{~nm}$ of $\mathrm{SiO}_{2}$, we observe a frequency shift of $190 \mathrm{MHz}$ for the first peak of the SMR and $150 \mathrm{MHz}$ for its second peak. These shifts include the temperature effects. For the QCM we get a $6.5 \mathrm{kHz}$ shift. The mass sensitivities shown by our SMRs are in the range of 2000 $\mathrm{Hz} \mathrm{cm} / \mathrm{ng}$ and their TCFs in the range of $+20 \mathrm{ppm} /{ }^{\circ} \mathrm{C}$ up to $+30 \mathrm{ppm} /{ }^{\circ} \mathrm{C}$. These values can vary (higher mass sensitivities and lower TCFs) depending on the device design and the materials deposited on top in a predictive way. The mass sensitives shown by the SMRs are three orders of magnitude higher than those of the QCM. SMRs achieve a mass resolution in the fg range, whereas the best resolution of commercial QCMs lies in the range of pg. If we additionally consider the smaller surface area of the devices, SMRs respond faster upon smaller amounts of deposited mass.

\section{CONCLUSIONS}

We have presented initial results of SMR-based devices being used as thin film monitoring sensors in a PVD system. They have been tested together with a commercial $6 \mathrm{MHz}$ QCM. SMRs show three orders of magnitude higher mass sensitivities and, additionally, our specifically designed SMRs can discriminate the temperature effect without the need of a reference sensor or of a cooling system, as QCMs do. SMRs work at higher frequencies with the inherent law of noise scaled up with frequency. Nonetheless, the evolution of the vector network analyzers market offers nowadays smaller, portable and high performance solutions with lower prices. This allows a new way of testing acoustic sensors, particularly at high frequency, achieving very fast data acquisition with a higher resolution level limited by the frequency stability in the few $\mathrm{kHz}$ range. With such limits of detection SMRs can achieve absolute resolutions of fg. The readout electronics offered currently on the market for QCMs permit pg resolutions using the best and most expensive option. All in all, together with the array option offered by SMRs, we can consider them as highly promising substitutes of QCMs in the new generations of deposition systems.

\section{ACKNOWLEDGMENT}

The authors would like to acknowledge Prof. Enrique Iborra, co-founder of Sorex Sensors Ltd, for his priceless guidance and support.

\section{REFERENCES}

[1] G. Sauerbrey, "Verwendung von Schwingquarzen zur Wagungdiinner Schichten und zur Mikrowagung," Zeitschrift fur Phys., vol. 155, pp. 206-222, 1959.

[2] X.-H. Huang, W. Pan, J.-G. Hu, and Q.-S. Bai, "The Exploration and Confirmation of the Maximum Mass Sensitivity of Quartz Crystal Microbalance," IEEE Trans. Ultrason. Ferroelectr. Freq. Control, vol. 65, no. 10, pp. 1888-1892, Oct. 2018.

[3] "IC6 Thin Film Deposition Controller - INFICON." [Online]. Available: https://products.inficon.com/en-us/nav-products/product/detail/ic6/.

[4] "VCSEL Market by Material (GaAs, InP, GaN), Type (Single Mode and Multimode), Application (Data Communication, Sensing, IR Illumination, Pumping, Industrial Heating, and Emerging Applications), End User, and Geography - Global Forecast to 2023." [Online]. Available:

https://www.researchandmarkets.com/research $/ 2 \mathrm{hm} 9 \mathrm{w} 9 / \mathrm{vcsel}$ ? $=12$.

[5] R. Gabl, E. Green, M. Schreiter, H. D. Feucht, H. Zeininger, R. Primig, D. Pitzer, G. Eckstein, and W. Wersing, "Novel integrated FBAR sensors: a universal technology platform for bio- and gas-detection," Proc. IEEE Sensors 2003 (IEEE Cat. No.03CH37498), vol. 2, pp. 1184-1188, 2003.

[6] R. P. O'Toole, S. G. Burns, G. J. Bastiaans, and M. D. Porter, "Thin aluminum nitride film resonators: miniaturized high sensitivity mass sensors," Anal. Chem., vol. 64, no. 11, pp. 1289-1294, Jun. 1992.

[7] T. Mirea, J. Olivares, M. Clement, J. L. Olivera, J. Sangrador, and E. Iborra, "AlN-solidly mounted resonators sustaining up to $1000^{\circ} \mathrm{C}$ with TCF compensation," in 2017 Joint Conference of the European Frequency and Time Forum and IEEE International Frequency Control Symposium, EFTF/IFC 2017 - Proceedings, 2017. 